\title{
Balkanologie
}

Balkanologie Revue d'études pluridisciplinaires

Vol. VII, $n^{\circ} 2 \mid 2003$

Volume VII Numéro 2

\section{De la langue intime à une langue étrangère : la langue allemande en Croatie dans la première moitié du XIX ${ }^{e}$ siècle}

L'exemple du journal de Caroline/Dragojla Jarnević

\section{Daniel Baric}

\section{OpenEdition}

\section{Journals}

Édition électronique

URL : http://journals.openedition.org/balkanologie/490

DOI : $10.4000 /$ balkanologie.490

ISSN : 1965-0582

Éditeur

Association française d'études sur les Balkans (Afebalk)

Édition imprimée

Date de publication : 1 décembre 2003

Pagination : 9-18

ISSN : 1279-7952

\section{Référence électronique}

Daniel Baric, « De la langue intime à une langue étrangère : la langue allemande en Croatie dans la première moitié du xIx ${ }^{\mathrm{e}}$ siècle », Balkanologie [En ligne], Vol. VII, nº 2 | 2003, mis en ligne le 19 février 2009, consulté le 17 décembre 2020. URL : http://journals.openedition.org/balkanologie/490 ; DOI https://doi.org/10.4000/balkanologie.490 


\title{
DE LA LANGUE INTIME À UNE LANGUE ÉTRANGÈRE : LA LANGUE ALLEMANDE EN CROATIE DANS LA PREMIÈRE MOITIÉ DU XIX' SIÈCLE. L'EXEMPLE DU JOURNAL DE CAROLINE/DRAGOJLA JARNEVIĆ
}

\author{
Daniel Baric*
}

L'objet de ce texte est de faire part d'une approche possible de l'appartenance à l'Empire des Habsbourg dans une zone périphérique. Il s'agit plus précisément d'une tentative d'y définir la place que prenait la langue allemande, langue dominante, utilisée dans tous les centres de décision de l'Empire, même au niveau municipal. Le rôle des langues dans le système habsbourgeois n'a pas échappé aux historiens des pays issus de l'Empire ${ }^{1}$. Mais l'étude de la langue se présente habituellement comme le récit d'une reconquête contre des forces hostiles à la nation ${ }^{2}$. Dans cette perspective, écrire l'histoire des langues nationales c'est écrire l'histoire du point de vue des vainqueurs. Et dans un tel cas, il est inévitable que l'on occulte l'autre partenaire, la langue allemande, dominante jusqu'au $\mathrm{XIX}^{\mathrm{e}}$ siècle dans le champ culturel et politique et qui sera évincée progressivement durant la deuxième moitié du $\mathrm{XIX}^{\mathrm{e}}$ siècle pour disparaître peu à peu au $\mathrm{XX}^{\mathrm{e}}$ siècle.

Or, il paraît justifié de ne pas éliminer trop vite la question de la résistance de la langue allemande face à l'avènement des langues "nationales". La place de l'allemand dans la société des régions non peuplées exclusivement de germanophones (en l'occurrence, dans les frontières du Royaume de Hongrie) est

\footnotetext{
- ATER à l'Université du Havre.

${ }^{1}$ Ceci est vrai dès le XIX ${ }^{e}$ siècle, cf. Gumplowicz (Ludwig), Das Recht der Nationalitäten und Sprachen in Österreich-Ungarn, Innsbruck, 1879 ; plus récemment, Wandruszka et Urbanitsch ont fait un tour d'horizon de la question chez les différents peuples de la monarchie (Wandruszka (Adam), Urbanitsch (Peter), eds., Die Völker des Reiches, Vienne, 1980). En français, on pourra lire un chapitre du livre de Michel (Bernard), Nations et nationalismes en Europe centrale XIX ${ }^{e}-X X^{e}$ siècle, Paris : Aubier Montaigne, 1995, pp. 31-64, consacré à la question de la langue (" L'imaginaire de la langue ").

${ }^{2}$ Pour une introduction à cette thématique, on peut consulter : Caussat (Pierre), Adamski (Dariusz), Crépon (Marc), La Langue source de la nation, Sprimont : Mardaga, 1996.
} 
le signe d'un lien particulier avec le cœur politique de l'Empire. À ce titre, il semble légitime de retracer l'évolution de sa place, comme marqueur d'une cohérence de l'Empire dans ses couches dirigeantes.

Le biais proposé ici est particulier : il n'est pas question de partir de généralités, mais du particulier. C'est à partir d'un cas que l'on pourrait tenter de voir ce qu'il en est du rapport à l'Empire à travers la pratique et l'attachement à la langue allemande.

Il sera question ici d'une femme croate, qui, par la documentation qu'elle a laissée, permet d'examiner la question. Caroline Jarnević a en effet écrit un journal intime de 1833 (à partir de ses 21 ans) jusqu'à la fin de sa vie en $1874^{3}$. Ce journal, tenu intialement en allemand, sera rédigé en croate à partir des années 1840 . En cela, il reflète bien l'essor des langues nationales. Mais l'attrait de ces pages est qu'elles permettent de suivre, pratiquement au jour le jour, l'emprise grandissante du croate sur l'allemand, ou bien peut-être, ceci est à voir, la persistance de l'allemand sous le croate.

\section{LE MODÈLE/MOULE ALLEMAND}

Caroline Jarnević est née en Croatie occidentale, à Karlovac, en 1812, d'un père commerçant. Elle évolue tant qu'il est vivant dans un milieu relativement aisé4. Les choses commencent à devenir difficiles à partir du décès du père, alors qu'elle est encore jeune. Sa vie est dès lors marquée par la nécessité de trouver des sources de revenus; son journal témoigne de sa recherche constante de revenus suffisants : elle est tour à tour couturière, modiste, gouvernante, enseignante ; et ces activités lui prennent le temps qu'elle aimerait consacrer à la lecture et à l'écriture. C'est durant l'enfance qu'elle s'imprègne de culture allemande. Elle fréquente peu les écoles car elles sont rares, mais tout ce qui était enseigné l'était en allemands.

" En matière de travaux manuels, notre institutrice recevait de Vienne divers dessins et modèles, qui formaient ensuite la base de nos travaux. " ${ }^{6} \grave{A}$ tra-

\footnotetext{
3 Ce qui en fait une femme de son temps, comme le montre la périodisation du journal intime en France effectuée par P. Lejeune (Lejeune (Philippe), Le moi des demoiselles. Enquête sur le journal de jeune fille, Paris : Seuil, 1993). C'est seulement au début de la monarchie de Juillet que la pratique semble s'établir et se répandre (p.422) avec des " dominantes n: 1830-1850, période " romantique ", 1850-1880, journal " ordre moral ", ensuite journal "Troisième République " (p. 65). La tonalité des écritures ordinaires et intimes semble donc étroitement liée aux cadres politiques généraux.
}

4 Pour une esquisse biographique, cf Cuvaj(Antun), Gradja za povijest školstva, Zagreb, 1910, IV, pp. 63-69 ; Luksic (Irena), " postface ", in Jarnević (Dragojla), Dnevnik, Karlovac : Matica Hrvatska, 2000.

\footnotetext{
5 Jarnević (Dragojla), op. cit., p. 9
}

6 Ibid. 
vers ce genre d'activités, elle rentre en contact avec l'univers des autres petites filles de tout l'Empire, qui eurent leur éducation au travail, l'éducation au goût formées sur un modèle viennois.

L'enfance est un moment de lectures désordonnées, solitaires : romans, livres de médecine, récits de voyage, " le chaos s'installa dans mon esprit, et personne ne se préoccupait du développement de la jeune fille que j'étais et de son avenir. Ces Ritter und Geistergeschichten [Histoires de chevaliers et de fantômes] me coûtèrent bien des nuits blanches $n$.

Elle se fait plus précise, lorsque, parvenue à l'âge adulte, elle revient sur ses premières lectures : " Lafontaine, Kotzebue, Schilling et Schiller, Kramer et Pichler, Frohberg, la Motte Fouqué, Clauren, W. Scott, Cooper, Körner, Uhland, Byron et les autres ${ }^{8}:$ le programme est bien autrichien, dépendant de ce qu'il y a dans les librairies et la seule bibliothèque de prêt de la ville 9 . Le retour critique sur la littérature "romantique" et les faiblesses du réseau autrichien de bibliothèques, de librairies et d'une manière générale de la circulation des livres est un thème que l'on retrouve dans un autre texte autobiographique évoquant Karlovac à la même époque ${ }^{10}$ la quête du bon livre, du livre scientifique en particulier (botanique, géographique), aboutit soit à une détestation du système metternichien puis habsbourgeois dans son ensemble (Tkalac), ou bien à une affirmation de l'identité croate face à la culture de langue allemande, où l'Empire d'Autriche ne semble pas perçu de manière autonome (Caroline Jarnević). Il est en tout cas indéniable que dans les années de formation, les enfants de Karlovac baignent dans un milieu germanophone dès lors qu'il s'agit du monde scolaire. Cette imprégnation ne va pas sans contradictions pour des enfants issus de familles croates, pour lesquelles l'acculturation au monde urbain et donc germanophone n'est que récent, et encore imparfait. "Jusqu'à l'âge de 18 ans, (...) j'ai remarqué que les Croates ont un niveau de culture bas, et qu'ils ont un naturel simple. Que seuls les avocats et les religieux, à quelques exceptions près, sont des gens qui ont des connaissances, et que les autres sont de faibles esprits, comme l'était le premier qui me demanda en mariage. $n^{11}$

Ainsi, en recevant une éducation semblable à celle des autres jeunes filles de l'Empire, mais à un endroit où cette éducation n'est pas partagée par tous, la jeune fille se sent en décalage. " Pour calmer mes sens bouleversés, mon

\footnotetext{
${ }^{7}$ Ibid., p. 10.

${ }^{8}$ Ibid., p. 85 .

9 La situation de la lecture en Croatie est présentée par Kessler (Wolfgang), « Buchproduktion und Lektüre in Zivilkroatien und-slawonien zwischen Aufklärung und nationaler Wiedergeburt n, Archiv für Geschichte das Buchwesens, 16, 1977.
}

10 Tkalac (Emmerich), Jugenderinnerungen aus Kroatien, Leipzig, 1894.

${ }^{11}$ Jarnević (Dragojla), op. cit., p. 11. 
cœur éploré, je me suis lancée dans l'écriture, et ainsi prirent naissance sous ma plume des Fantazien eines gequälten Herzens [fantaisies d'un cœur torturé] et d'autres choses encore. "12 L'écriture - en allemand - est pour elle un refuge face au monde qui l'entoure, qui ne correspond pas aux univers dont elle prend connaissance à travers ses lectures allemandes.

Les années de jeunesse sont marquées par des liens avec cette langue. L'attachement à une personne passe ainsi chez elle par une prise de conscience que la langue peut être plus ou moins bien maniée. Ainsi rend-elle compte d'une rencontre émerveillée avec un Autrichien, Franjo [Franz] Redinger, originaire de Brno en Moravie, employé dans une imprimerie de la ville : " comme il parle bien allemand ! Jusqu'à ce soir je n'avais pas encore entendu parler allemand, alors que je n'arrête pas de me servir de l'allemand [en société] ", " chaque mot [était] à sa place "; " il a passé deux ans en Prusse ", "Quelle discussion cela fut ! O bien différente de celles que les gens ont à Karlovac. Mots choisis, finesse des expressions (...) $n^{13}$.

Puis, quelques mois plus tard :

depuis que je connais Redinger,j'ai remarqué la différence entre les Croates et un homme allemand [njemackoga roda] cultivé. Certes, le Croate est d'une nature bienveillante, un diamant qui n'a pas été taillé, mais j'ai horreur de la grossièreté qui émane de la plupart d'entre eux, et pour cette raison je ne trouve pas dans les Croates d'amis avec lesquels je pourrais m'amuser et encore moins aimer. ${ }^{14}$

Tel Croate souffre du contraste : Jovan Ferric, de Slavonie : " il parle si mal allemand que je ne peux m'empêcher de rire en l'écoutant, et il est maladroit dans ses gestes à un point incroyable "15. À ce moment-là de sa vie, Caroline Jarnević perçoit sur le même plan et juge de la qualité d'une personne à son maniement de l'allemand, qui est mis en relation avec son comportement général, et il est un fait que les deux semblent aller de pair. Un allemand défectueux est pour elle la preuve d'une éducation et d'un esprit insuffisants.

Car jusque dans les années 1830, le journal, tenu en allemand, témoigne d'une soif d'apprendre, d'une envie d'ouverture au monde qui ne peuvent avoir de sens que dans une éducation en allemand, qui donne la possibilité d'avoir accès au monde au-delà des frontières du monde germanique. Ainsi note-t-elle les livres qui lui permettent d'accéder à de vastes connaissances, tel un dictionnnaire des mots étrangers acheté en 1836 (Wörterbuch von 3000 Fremdwörtern). On retrouve dans l'écho de ses lectures une délectation à suivre les élans de Schiller, mais la conscience que Goethe donne des bases solides à

\footnotetext{
${ }^{12}$ Ibid., p. 12.

${ }^{13}$ Ibid., p. 22 (27 avril 1833).

${ }^{14}$ Ibid., p. 43 (septembre 1833).

15 Ibid., p. 57 (janvier 1834).
} 
la connaissance ${ }^{16}$. Le monde entier n'est alors accessible que par l'allemand pour Caroline Jarnević, et Karlovac est lié par cette langue au reste du monde.

\section{LE DÉTACHEMENT}

En 1836 apparaissent les premières manifestations du mouvement illyrien, précurseur du mouvement identitaire national croate ; c'est avec étonnement qu'elle enregistre dans son journal que ses voisins se mettent à vouloir parler croate, alors qu'ils ne parlaient qu'allemand avec elle : " ils parlent d'un certain illyrisme et veulent convaincre ma sœur et moi de renoncer à l'allemand. Comment renoncerais-je à l'allemand, alors que je ne parle pas croate ; je veux dire suffisamment pour pouvoir communiquer " ; " je n'ai pas de mots pour mes pensées et mes sentiments " en croate ${ }^{17}$; " Schiller, Goethe et Körner me sont plus chers que tout l'illyrisme $n^{18}$ note-telle la même année.

Cependant, c'est durant cet été-là qu'elle se rend à Ozalj, château situé dans les environs de Karlovac. Elle pense là-bas à ceux qui ont défendu la patrie (domovina), en particulier Zrinski et Frankopan. On assiste alors à la naissance d'un thème, repris dans les nouvelles qu'elle écrira et publiera en croate, quelques années plus tard. Mais pour l'heure elle pense en allemand, transcrit en allemand dans la ville de garnison de Karlovac, ville à la frontière des Confins militaires, face à la Bosnie ottomane, son exaltation à l'évocation de figures historiques condamnées à mort par les Habsbourg au XVII ${ }^{e}$ siècle pour avoir trahi la dynastie et pris contact avec les Ottomans au nom de la défense des intérêts croates (ou du moins est-ce la version qu'elle retient de leur condamnation) ${ }^{19}$. Le fossé commence à se creuser entre la culture qui l'a formée et ses émotions nationales naissantes.

En 1837, le ton se fait plus conciliant à l'égard de la langue croate.

J'ai du mal à accepter ce nom d'Illyrien, d'Illyrienne - au lieu de Croate - mais puisque des gens savants comme un certain Gaj à Zagreb et d'autres encore /en ont décidé ainsi/ [...] je pense /aussi/ qu'il doit en être ainsi. Sauf que je n'y arrive pas avec cette langue illyrienne, ou croate, et il y eut pas mal de rire lorsque ces jeunes gens me forcèrent à parler croate..$^{20}$

\footnotetext{
${ }^{16}$ Ibid., p. 77

${ }^{17}$ Ibid., p. 78

${ }^{18} \mathrm{Ibid}$., p. 80

19 Ibid., p. 74 (20 juin 1836).

${ }^{20}$ Ibid., p. 97 (1837).
} 
Certes, il y a encore de la résistance à abandonner l'allemand : " j'ai écrit une petite nouvelle en allemand. J'ai essayé d'écrire en croate, mais ça ne va pas. Je n'arrive vraiment à rien penser en croate, et a fortiori à écrire $n^{21}$.

Mais le véritable tournant est opéré lors d'un séjour de plusieurs mois à l'étranger. À Graz, Trieste et Venise, où elle peut pourtant communiquer aussi bien en allemand qu'en italien (appris à Karlovac), elle découvre qu'elle a envie d'entendre la langue du pays laissé derrière elle. Elle a l'expérience d'un détachement de la culture de langue allemande. En vivant avec les représentants de la culture de langue allemande, elle comprend à quel point la vie calme à Karlovac est différente de celle de Graz, pleine de tentations. On lui demande d'où elle vient, son dialecte est jugé "beau", sa manière de parler et ses expressions choisies : elle est tout de suite perçue comme non Styrienne $e^{22}$.

Mais la grande tâche qu'elle se donne à Graz, c'est l'apprentissage systématique du croate à partir d'un dictionaire dalmate-allemand-italien, avec le but donné par ses (jeunes) enseignants, Croates séjournant à Graz, de produire de la littérature en croate ${ }^{23}$. Sa richesse, écrit-elle, c'est sa langue maternelle : " mes efforts sont assez grands pour me l'approprier, mais cela n'est pas si facile, puisque j'ai la langue allemande dans le sang "24. Sa perception d'un processus physiologique à l'œuvre en elle montre la difficulté qu'elle ressent à changer de langue, ce qui revient à changer de substance, de corps. À Venise, quelques mois plus tard, elle écrit : " il y a quelque chose en moi qui ne se laisse pas éloigner de la patrie, comme je le voulais $n^{25}$ : les mois à l'étranger sont des moments d'oscillation entre deux pôles, deux langues, qui ont des difficultés à cohabiter en elle.

Les moments de doute ne manquent pas, en particulier lorsqu'elle pense qu'elle a commencé déjà relativement âgée, et que c'est une tentative folle de vouloir s'approprier le croate et créer en croate ${ }^{26}$. " Je passe d'un travail à un autre, j'écris, je lis, je fais la leçon aux enfants, et je couds lorsque j'en ai le loisir. (...) On me nomme "la rigorosa Tedeska" (...) mais je demande à la comtesse qu'elle ne dise à personne que je suis Allemande, car je ne veux pas l'être, je suis Croate, comme je le dis à tous lorsque j'en ai l'occasion. $n^{27}$

Mais dans le processus d'apprentissage de la langue croate, l'allemand est la langue de référence. C'est à partir de termes allemands que son professeur,

\footnotetext{
${ }^{21}$ Ibid., p. 105 (20 mai 1838).

${ }^{22}$ Ibid., p. 117 (juin 1839).

23 Ibid., p. 121.

24 Ibid., p. 126.

25 Ibid., p. 151 (en 1840, à Venise).

${ }^{26}$ Ibid., p. 172 (15 octobre 1840).

${ }^{27}$ Ibid., p. 150 (30 mars 1840).
} 
Trnski, introduit dans sa correspondance avec elle des termes croates inconnus $^{28}$.

Et après plusieurs années de travail acharné, Caroline Jarnević cesse d'écrire, et c'est Dragojla qui prend la plume. Le changement de langue se répercute aussi sur le prénom, slavisé, en prenant en compte l'étymologie. À partir de 1841, Dragojla Jarnević écrit en croate et poursuit sa trajectoire croate, qui devrait l'éloigner de plus en plus de l'enfance et de la jeunesse autrichienne de langue allemande.

En 1848 , la rupture semble complètement consommée. À l'été 1848 , le représentant des Croates, Jelačić est accueilli en libérateur à Karlovac. Dragojla remarque de ridicules jeunes filles qui essayent de parler allemand, mais qui n'ont pas la bonne prononciation, ni ne savent parler de manière élégante; le soir, un bal serbe est organisé : mais les discussions se font toujours en allemand, jusqu'au moment où tout le monde passe à la langue nationale. Alors que Dragojla maîtrise l'allemand, elle semble ne pas vouloir prêter allégeance à la dynastie des Habsbourg, en 1848, comme durant toute la décennie qui précède. Et ce sont les jeunes filles d'une nouvelle génération, qui souhaiteraient pourtant marquer leur soutien envers Jelačić et donc envers Vienne, qui ne parlent plus l'allemand comme des germanophones. À Karlovac, l'allemand est devenu une langue étrangère, apprise, mais qui n'est plus la langue de la communication la plus aisée ${ }^{29}$.

\section{RÉMANENCES : L'OUBLI IMPOSSIBLE}

Le journal tenu en croate est-il pourtant exempt de traces du passé allemand ? Rien n'est moins sûr. On ne cesse, dans les pages qui couvrent les décennies qui suivent le passage au croate, de trouver des "buttes témoin", des retours de l'allemand. Ainsi, à la fin de la première année d'écriture en croate, au 31 décembre, une longue page témoigne d'une envie de s'épancher dans cette langue, "à jamais chère " [in der mir ewig theueren Sprache]. Le bilan de cette année de travail d'écriture en croate semble ne pouvoir être fait qu'en allemand. Comment dire sinon en allemand la douleur d'être rentrée en Croatie ? Certes, on lui conseille d'écrire en croate ; et une telle suggestion vient du responsable de l'éducation dans la province, Frass, haut fonctionnaire autrichien et germanophone, qui au contact des populations dans les Confins mili-

\footnotetext{
${ }^{28}$ Lettre de Trnski, 1839, écrite de Graz (in Srepel (Milivoj), ed., Gradja za povijest književnosti, Zagreb : JAZU, vol. 3, 1901, p. 180) : " car je ne connais d'amour de la patrie sans vertu (...) une âme perfide et dénuée de vertu de peut rien sentir de noble. "
}

${ }^{29}$ Jarnević (Dragojla), op. cit., p. 345 (20 août 1848). 
taires s'est persuadé qu'une éducation et une culture ne peuvent réellement se développer que dans la langue du peuple. "Ma patrie a besoin de culture [Ausbildung], de littérature et de gens de lettre qui écrivent en langue nationale. ${ }^{30} \mathrm{~A}$ travers la réflexion de Dragojla Jarnević, on perçoit aussi le penchant d'une certaine politique hasbourgeoise : le désir de transfert d'une éducation autrichienne en langue nationale. À ce titre la position autrichienne se singularise face au système d'éducation d'autres empires : prussien, français, ou vénitien, où la langue nationale des peuples minoritaires n'est pas en position d'espérer jouer un véritable rôle dans l'éducation.

Dans le texte du journal, la langue allemande informe la strate croate, ce qui en rend la lecture difficile aujourd'hui. La longue histoire de l'édition complète est une preuve de la situation intermédiaire de ce texte, qui n'a pas pu être donné à la lecture avant l'an 200031. La réception critique de Dragojla Jarnević ne s'est pas située de plein pied dans le champ littéraire, la critique a toujours mis en avant les défauts de la langue croate (par mimétisme avec la perception qu'elle-même avait de sa langue croate) ; or, cette langue croate est le reflet de l'état des relations entre l'allemand et le croate à un moment donné : au cours des mois et des années, on constate des fluctuations, des hybridations. Des pages entières, quelques lignes, des vers de Schiller qui remontent à la surface au moment d'une intense émotion, des mots chuchotés dans l'intimité témoignent de la prégnance du passé germanophone. Des mots allemands isolés continuent de ponctuer l'écriture ${ }^{32}$. Certains sont identifiés comme allemands, d'autres sont cachés, ils n'apparaissent que lorsque l'on fait ressortir dans les mots croates le calque allemand 33 . D'autres encore apparaissent après les mots croates pour les expliciter 34 .

Lorsqu'à soixante ans, elle décide de traduire elle-même les années du journal tenu en allemand, elle sait qu'elle doit se confronter avec une part d'elle-même qu'elle a essayé d'annihiler :

bien sûr, j'écrivais en allemand, car je considérais alors que l'allemand était ma langue maternelle. Et le fait qu'à soixante ans je décidai de traduire le journal en croate est l'occasion pour moi de remarquer qu'il y a bien de la " fantaisie " dans

\footnotetext{
30 Ibid., p. 184 (retour fin 1841). de la Matica hrvatska de Karlovac.

32 Jarnević (Dragojla), op. cit., p. 191 (niti mojih /Kunden/).

33 Ibid., p. 26 (" skroz i skroz je on vrlina " [durch und durch]).

34 Ibid., p. 312 (ćtliv /empfindsam, empfindelnd/).
}

${ }^{31}$ En 1957, des extraits avaient été donnés (Dvorzak (Stanko), 1913 Karlovački portreti, Zagreb, 1957), mais dans une langue adaptée au standard en vigueur un siècle après l'écriture du texte original ; et en 1985 , une étude assortie d'extraits avait été publiée (Zečević, Dragojla Jarnević, Zagreb, 1985), mais pour une édition "complète", dans la langue originale, avec les mots allemands résiduels, il fallut attendre l'édition 
ma vie, que je me propose de réduire à de plus justes proportions, et qu'il y a bien des fautes de grammaire dans le croate dans lequel j'ai commencé à écrire à partir de 1841.35

J'ai décidé de traduire toutes les années où j'ai écrit mon journal en allemand. Je ne sais si j'arriverai à achever ce travail, mais en tout cas, je m'y efforcerai. (...) Cela sera douloureux, j'aurai mal au cœur, mais je préfère qu'il en soit ainsi plutôt qu'il n'arrive en allemand dans les mains des Croates. Ce que j'ai écrit après 1841 en croate est vraiment une drôle de langue, car elle m'a trahie souvent; mais je ne peux réparer cela. Qu'elle subsiste comme une preuve de mes progrès dans la langue maternelle, et en même temps comme une preuve de mes peines et de ma lutte avec la faiblesse [de mes connaissances en croate]. ${ }^{36}$

Passer d'une langue à une autre, de manière volontaire, n'a donc pas suffit à marquer une séparation définitive avec ce qui fut une langue maternelle. Le journal de Caroline devenue Dragojla Jarnević est le témoignage de l'impossibilité, pour cette femme, à ce moment de l'histoire de l'Empire habsbourgeois, de se défaire complètement des structures linguistiques, culturelles dominantes.

\section{CONCLUSION}

Sans doute le journal de Caroline/Dragojla Jarnević est-il un document exceptionnel par sa longueur, sa richesse. Mais d'autres journaux intimes existent, publiés ou inédits, et devraient permettre, s'ils sont analysés sous l'angle du rejet et de la permanence de la culture allemande, de mettre en évidence des traces similaires d'hybridation : Oriovčanin $37, \operatorname{Vrhovac}^{38}, \ldots$ montrent à leur manière cette évolution.

Du reste, l'ambivalence à l'égard de la culture dominante de l'Empire habsbourgeois dans les régions formant autrefois l'Empire persiste bien après l'écriture du journal de Dragojla Jarnević, jusqu'au XX $\mathrm{XX}^{\mathrm{e}}$ siècle on en trouve des traces dans les œuvres littéraires, comme c'est le cas pour Krleža. L'apparition de mots et d'expressions allemandes est alors une prise de position face à

35 Ibid., p. 14 .

${ }^{36}$ Ibid., p. 71 (pour Zečević, op. cit., p. 39, la traduction est une prise de conscience que le journal est un genre littéraire, qu'il faut donc prendre en considération l'existence de lecteurs futurs, qui seront dans son esprit nécessairement plus à même de lire le croate que l'allemand).

37 Arhiv HAZU.

$3^{8}$ Publié en partie uniquement. 
l'Empire habsbourgeois. Chez Krleža, il y a variation depuis le rejet jusqu'à la nostalgie.

$\mathrm{Au} \mathrm{XIX}{ }^{\mathrm{e}}$ siècle cependant, vient un moment - dans les années 1830 dans la vie de Dragojla Jarnević - où parler allemand pose la question de la filiation. Parler, réfléchir, lire, se cultiver en allemand devient problématique, c'est désormais une trahison, un acte politique. Le journal intime, celui de Dragojla Jarnević, est un des reflets de cette inflexion. La ligne de fracture entre les langues apparaît dans l'écrit, sur toute une vie, et à ce titre le journal intime est un document très riche. Mais pour l'immense majorité des personnes, le passage d'une langue à une autre au cours d'une vie n'est pas documenté, et sans doute a-t-il existé selon des modalités très diverses. En chaque habitant de l'Empire s'est joué d'une manière particulière l'agonie de la langue dominante. 